\title{
BINARY FREQUENCY AMONG PRE-MAIN SEQUENCE STARS IN TAURUS AND OPHIUCHUS
}

\author{
M. Simon \\ Astronomy Program \\ State University of New York at Stony Brook \\ Stony Brook, N.Y. 11794, U.S.A.
}

\begin{abstract}
The lunar occultation technique applied in the IR offers a powerful means of identifying binaries among obscured young stars. Our program has revealed binaries with separations from 1 to $100 \mathrm{AU}$ in the Taurus and Ophiuchus star forming regions to about $\mathrm{K}=9$ mag. To date, 29 objects have been observed; 6 were discovered to be binaries. The observed binary frequency is about half that expected from the binary statistics of a comparable sample of field stars. The discrepancy is probably attributable to our insensitivity to binary systems with secondary mass much less than that of the primary.
\end{abstract}

Binaries among young stars are being identified by optical spectroscopy (Mundt et al. 1983, Mathieu 1988), by IR speckle interferometry (e.g. Dyck et al. 1981, Chelli et al. 1988) and by lunar occultations in the infrared (Simon et al. 1987). In the infrared $K$ band the IR occultation technique offers a powerful means of identifying binaries among obscured young stars to $\mathrm{K} \sim 9$ mag and angular separations from 0.005 to $0.5 \mathrm{arc}$ sec. The lower limit is set by the signal to noise of the data and the instrumental effects that smooth the Fresnel pattern (Simon et al. 1987). The upper limit is set by the photometer aperture, typically 4 to 6 arc sec. One can observe only at the dark lunar limb.

The moon passes over both Taurus and Ophiuchus, two of the nearest star forming regions. Because the Taurus star forming region is so extensive, occultations of the young stars within it occur each year. The opportunities to observe occultations of the young stars in Ophiuchus are more limited. The most recent occultations occurred in 1985-6 and the next series will be in 1991-3.

We have to date observed 29 objects, 19 in Taurus and 10 in Ophiuchus. The systems discovered to be binaries are DF, FF, HQ, and Haro 6-8 in Taurus and SR-12 and ROX 31 in Ophiuchus. That DF Tau and Haro 6-8 are binaries with separations, in the projection of the occultations, of $\sim .011$ and $\sim .560$ arc sec respectively are recent findings; the other systems are described by Simon et al. 1987.

A central question is how the binary statistics of the young stars and field stars compare. For now, we consider the binaries in Taurus and Ophiuchus together; when the sample is larger, 
it will be possible to analyze the regions separately. The stars in our sample are of late spectral type. The F3 - G2 dwarfs studied by Abt (1983) are the most comparable field star sample. Abt's results are in terms of binary period. Assuming $1 \mathrm{M}_{\odot}$ total mass for each binary system and $150 \mathrm{pc}$ as representative distance to both star forming regions, our angular resolution range 0.005 to 0.5 arc sec corresponds to systems with periods in the range $10^{2}$ to $10^{6}$ days. Spectroscopic binaries tend to have periods shorter than 100 days. Speckle observations at $K$ have a resolution limit of $\sim .120$ arc sec, or in the spirit of these estimates, systems with periods exceeding $10^{5}$ days. The occultation technique nicely fills this gap. Using Abt's result for the $10^{2}$ to $10^{6}$ day range of periods, and correcting for both orbital projections and the distribution of occultation directions, we estimate that about $40 \%$ of the systems observed, or 12 out of 29 , should be binaries.

Our sample is still small but it seems that we are detecting a smaller number of binaries than expected. The reason may lie in the mass distribution of the secondaries. If it proves similar to that of the "bifurcation" sample identified by Abt (1983), then, as we approach the faint end of our flux limited sample, we will start to be insensitive to systems with secondary masses much less than that of the primary. Data for more systems, providing information on the observed flux ratios will help resolve the situation.

A thoroughly studied sample of a significant number of young star binaries will yield values of the fundamental stellar parameters, provide data essential to the determination of the initial mass function, and contribute to our understanding of the formation and evolution of multiple star systems. We look forward to progress in these areas in the next few years as the moon sweeps southward through Taurus and again across Ophiuchus.

I am grateful to J. Benson, W. Chen, R. Howell, A. Longmore, M. Smith, B. Wilking, and G. Wright for observing occultations for this program and thank $W$. Chen and D. Peterson for discussions and help. This research was supported in part by NSF Grant AST 85-14337.

\section{References}

Abt, H.A. 1983, Ann. Rev. Astr. Ap., 21, 343.

Chelli, A. et al. 1988, Astr.Ap., in press.

Dyck, H.M. et al. 1982, Ap.J.Lett., 255, L103.

Mathieu, R.D. 1988, these proceedings.

Mundt, R. et al. 1983, Ap.J., 269, 229.

Simon, M. et al. 1987, Ap.J., 320, 344. 